\title{
Lidil
}

Revue de linguistique et de didactique des langues

$48 \mid 2013$

L'émotion et l'apprentissage des langues

\section{Gérer la charge émotionnelle liée à la langue arabe : un défi pour le professeur de langue étrangère}

Catherine Pinon

\section{OpenEdition}

Journals

Édition électronique

URL : http://journals.openedition.org/lidil/3321

DOI : $10.4000 /$ lidil.3321

ISSN : 1960-6052

Éditeur

UGA Éditions/Université Grenoble Alpes

\section{Édition imprimée}

Date de publication : 1 novembre 2013

Pagination : 115-135

ISBN : 978-2-84310-260-8

ISSN : $1146-6480$

Référence électronique

Catherine Pinon, «Gérer la charge émotionnelle liée à la langue arabe : un défi pour le professeur de langue étrangère », Lidil [En ligne], 48 | 2013, mis en ligne le 01 mai 2015, consulté le 19 avril 2019. URL : http://journals.openedition.org/lidil/3321 ; DOI : 10.4000/lidil.3321 


\title{
Gérer la charge émotionnelle liée à la langue arabe : un défi pour le professeur de langue étrangère*
}

\author{
Catherine Pinon**
}

\begin{abstract}
RÉsumé
Cet article s'intéresse aux différentes charges émotives liées à la langue arabe et à leur impact sur l'enseignement de cette langue vivante étrangère. Nous y décrivons les émotions que ressentent les élèves et qui peuvent rendre son apprentissage difficile et problématique. Nous expliquons certaines des situations propices à l'abandon de cette langue par les élèves, notamment en nous référant aux travaux de Billiez (1985, 2002) sur les pratiques langagières des descendants de migrants et aux statistiques de Filhon $(2002,2009)$ ainsi qu'à des réflexions antérieures (Pinon, 2011, 2012). Nous replaçons l'enseignement de l'arabe dans le contexte social actuel et voyons comment les représentations de la langue arabe, le rôle qu'elle joue comme marqueur identitaire et véhicule de l'islam entrainent parfois tout un processus de rejet de l'apprentissage de cette langue. Nous proposons donc une analyse de ce processus pour réfléchir à une démarche où, prenant conscience de certains phénomènes liés aux réactions que suscite la langue arabe, l'on s'efforce de comprendre et de gérer ces situations.
\end{abstract}

\section{ABSTRACT}

This paper deals with the various emotional charges of the Arabic language and their impact in the teaching of this foreign language. I will describe the emotions felt by the pupils that contribute to making it a problematic language, one that is difficult to learn. I'll explain some of the situations that result in a higher rate of giving up Arabic class with the help of works from Billiez $(1985,2002)$ on the language habits of the descendants of migrants and the statistics by Filhon (2002, 2009), and some of my previous works (Pinon, 2011, 2012). I will put

* Nous remercions les professeurs Driss al-Hachami et Frédéric Imbert pour leur relecture attentive.

** IREMAM. 
the teaching of the Arabic language in the current social context and see how the representations of the Arabic language, its role as a vector of identity and of the religion of Islam may sometimes trigger a process of rejection of learning it. I will offer an analysis of this process so as to think of a protocol in which being aware of some of the reactions in front of the Arabic language enables to understand and manage such situations.

L'enseignement de l'arabe littéral en France couvre tous les niveaux, depuis l'école élémentaire ${ }^{1}$ jusqu'au lycée et dans le supérieur ${ }^{2}$, s'inscrivant dans une longue tradition ${ }^{3}$. L'arabe est, objectivement, une langue vivante étrangère qui s'enseigne au même titre que les autres ${ }^{4}$. Mais force est de constater que le public d'apprenants et les situations réelles liées à cet enseignement en font une discipline particulière, qui est confrontée à des enjeux qu'ignorent souvent les professeurs d'autres langues. Il est souvent complexe d'enseigner l'arabe littéral ${ }^{5}$ : au quotidien, il nous faut adapter notre enseignement pour apprivoiser les réactions émotionnelles liées à cette langue, des réactions souvent extrêmes pouvant amener un bon nombre d'élèves à abandonner totalement l'apprentissage de l'arabe ${ }^{6}$. Cet article se base sur une expérience d'enseignement dans un lycée général de Lyon et une cité scolaire d'Avignon

1. À l'école élémentaire (enfants de 6 à 10 ans), par le biais de l'ELCO (Enseignement des langues et cultures d'origine) qui existe depuis 1973; dans le secondaire (de 11 à 14 ans pour le collège, de 15 à 18 ans pour le lycée) sous l'égide de l'Éducation nationale.

2. Une étude récente de la $15^{\mathrm{e}}$ section du Conseil national des universités donnait le chiffre de 6000 étudiants environ dans l'enseignement supérieur (communication privée) en 2011-2012.

3. L'agrégation d'arabe, qui existe depuis 1906, est la plus vieille agrégation de langue et couronne une longue tradition orientaliste. Voir MEN (2008).

4. En primaire, on enseigne surtout les dialectes arabes (langues maternelles) alors que dans le secondaire, on enseigne avant tout l'arabe littéral (langue officielle), tout en privilégiant à l'oral un registre moyen où les dialectes se mélangent à la variété standard.

5. Nous entendons par là l'arabe moderne, langue officielle des pays membres de la ligue arabe, qui n'est la langue maternelle de personne. On l'oppose souvent aux dialectes, les langues maternelles des Arabes.

6. Nous parlerons plus volontiers d'élèves que d'étudiants, car la plupart des cas concrets seront tirés d'expériences d'enseignement au collège et au lycée. La proportion des élèves à ce point en difficulté varie considérablement d'un établissement à un autre. 
entre 2007 et $2013^{7}$. Il a pour objet d'analyser le processus à l'origine du refus de l'apprentissage de la langue très fréquent de la part des élèves, pour proposer des réponses didactiques adéquates. Ces pistes de réflexions pourront donner lieu à une étude de cas plus systématique.

Ce que disent Billiez et Trimaille (2001) du terrain migratoire pour le sociologue peut s'appliquer à l'enseignement de l'arabe, à savoir que cela «nécessite une certaine passion, doublée d'une approche compréhensive [...], et oblige à sortir des cadres bien établis, tout en préservant un équilibre fragile entre engagement et distanciation» (p. 1). Nous souhaitons ici faire preuve de la lucidité et du recul nécessaire pour analyser une situation qui, au quotidien, provoque beaucoup d'émotions fortes et parfois contradictoires. Nous pouvons faire nôtre la définition que donne Livet de l'émotion, entendue comme des «réactions à la perception d'un différentiel» (Channouf \& Rouan, 2002, p. 58), différentiel qui s'évalue par rapport à nos attentes inconscientes. Cette définition nous amène à considérer que l'enseignant, tout comme l'élève, génère une charge émotionnelle supplémentaire qu'il doit apprendre à maitriser.

L'enseignement de l'arabe doit composer avec de nombreux facteurs émotionnels : certaines situations peuvent provoquer des émotions négatives telles que la colère, l'opposition ou le rejet, qui sont néfastes pour l'apprentissage; d'autres génèrent au contraire des émotions positives aidant l'élève dans son apprentissage, à l'instar du plaisir, de la joie ou de la reconnaissance. Le processus de rejet de l'arabe peut être schématisé ainsi :

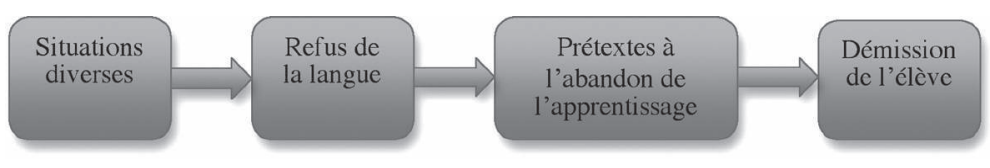

Ainsi, dans notre perspective, des situations différentes peuvent produire le même résultat : un refus d'apprendre l'arabe, qui aboutit à la démission de l'élève qui justifie cet abandon par différents prétextes. La part la plus importante de notre travail sera donc d'analyser ces différentes situations, mais aussi de repérer la manière avec laquelle ce refus

7. Sur environ 80 élèves, une seule était «franco-française», la quasi-totalité étant d'origine maghrébine. 
se manifeste en classe et les prétextes évoqués par les élèves démissionnaires pour justifier leur attitude vis-à-vis de la langue. À partir de ces analyses, nous proposerons des stratégies didactiques au sens large, à même de bloquer ce processus, et ce aux différents niveaux de cette chaine.

\section{1. État des lieux des situations propices au décrochage}

\subsection{L'arabe, une langue très chargée émotionnellement}

Les arabisants ne savent pas toujours par où commencer lorsqu'il s'agit de définir la langue arabe. Sous ce terme unique se cachent en effet des états et des variétés très diverses, puisqu'on dénombre notamment l'arabe du Coran, linguistiquement pré-classique; l'arabe classique (nous entendons par classique la langue rendue classique par les grammairiens arabes médiévaux; celui de la littérature médiévale); l'arabe moderne (une variété plus moderne de la première, langue officielle des États membres de la Ligue arabe, utilisée dans la littérature moderne et dans la presse); les vernaculaires qu'on nomme habituellement «dialectes». Avant de revenir sur les facteurs historiques, sociaux et sacromythologiques qui font de l'arabe une langue hautement symbolique, il convient de rappeler que l'arabe que nous enseignons, l'arabe littéral, n'est la langue maternelle de personne. Pour les élèves dialectophones ou pour ceux antérieurement scolarisés dans le monde arabe, on peut considérer qu'il s'agit d'une langue seconde, mais pour les autres, il s'agit bien d'une langue étrangère. Une langue étrangère qui n'est jamais ${ }^{8}$ parlée dans des situations de communication réelle.

\subsection{Sacralisation historique et sociale de l'arabe}

Les différentes valeurs dont est revêtue la langue arabe ont une influence considérable sur la perception qu'ont nos élèves de cette langue. C'est pourquoi il est nécessaire de revenir ici, quoique brièvement, sur la double sacralisation que connait la langue arabe (Pinon, 2012) : d'une part, une sacralisation théologique car l'arabe étant la langue du Coran, livre sacré des musulmans, l'évolution de la langue s'est poursuivie

8. Il convient de nuancer quelque peu ce propos. Deux arabophones parlant des dialectes où l'intercompréhension est faible peuvent, s'ils le connaissent tous les deux, être tentés d'utiliser l'arabe littéral pour communiquer. 
dans l'idée de dégradation d'un arabe pur, amenant les locuteurs des langues arabes vernaculaires à dénigrer ces dernières au profit d'un arabe fantasmé et investi d'une supériorité linguistique et stylistique mythique. Ensuite, une sacralisation idéologique s'est ajoutée à la première, au $\mathrm{Xx}^{\mathrm{e}}$ siècle, en faisant de cette langue l'outil majeur du nationalisme arabe.

Nos élèves ont en tête cette dualité axiologique qui les amène souvent à considérer l'arabe comme une langue inaccessible. Ce sentiment engendre un certain complexe vis-à-vis de la langue qui n'est pas propice à un apprentissage serein, surtout chez les élèves qui découvrent l'arabe à l'école. Cette attitude de culpabilisation linguistique, comme l'a nommée Billiez (1985), est exprimée par les locuteurs qui opposent le «vrai» arabe, celui appris à l'école, au «mélange» qui désigne leur véritable langue maternelle. On constate en fait que cette fracture entre le «vrai arabe» et la langue des immigrés en France est la même que celle qui oppose, de manière négative, les dialectes arabes à la langue appelée fush ${ }^{9}$ pour une très forte majorité des locuteurs arabes du monde arabe et qui a amené Calvet (1999) à développer le concept de schizoglossie de l'arabe.

Au-delà de ces considérations culturelles qui font de l'arabe une langue étrangère à part, c'est le lien avec un contexte social très particulier en France, celui de l'immigration maghrébine, qui doit être fait. Les travaux de Billiez (1985) ont montré à quel point les jeunes descendants de migrants investissaient la langue arabe d'une fonction symbolique fondamentale. Même pour ceux qui ne la parlent pas, elle tient une place centrale dans leur schéma de représentation linguistique. La langue des parents voire des grands-parents demeure un facteur d'identification pour ces jeunes souvent stigmatisés ${ }^{10}$. Il faut aussi rappeler que si $1 / 3$ des parents transmettent l'arabe pour préparer leurs enfants à un retour définitif au pays ${ }^{11}$, la principale raison est que la langue maternelle

9. Littéralement : la langue arabe «la plus éloquente », l'arabe classique.

10. Selon Billiez (1985), ce n'est pas le cas pour les descendants de migrants hispanophones, qui pratiquent, semble-t-il, beaucoup plus facilement l'espagnol en milieu familial et avec leurs pairs et le français dans les situations extra-familiales, sans l'insécurité linguistique que l'on décèle chez les arabophones.

11. Filhon (2009) estime à $31,1 \%$ les parents arabophones qui envisagent un retour définitif au pays (à 34,1\% les berbérophones), d'après l'étude de 1999. La situation a certainement changé depuis, mais ces chiffres renvoient à l'un 
est identifiée comme étant le vecteur de la mémoire familiale. Pour l'arabe, deux fonctions importantes sont assignées à la langue : celles d'un outil du savoir permettant d'accéder à l'enseignement dans le pays d'origine, à la presse et aux médias en général, aux enseignements religieux, et celle d'une valeur culturelle et culturelle, véhicule de l'arabité et de l'islam.

\subsection{Les pratiques linguistiques réelles de nos élèves}

\subsubsection{Langue familiale}

On considère souvent que ces élèves sont dialectophones : l'arabe enseigné, nous l'avons dit, n'est la langue maternelle de personne. Or, c'est de moins en moins le cas ${ }^{12}$ et il faut noter que l'arabe langue maternelle se perd de plus en plus en France. Selon les chiffres publiés par Héran, Filhon et Deprez (2002), moins de la moitié des parents transmettent en France l'arabe à leurs enfants. En réalité, aucune étude à grande échelle ne s'est intéressée aux pratiques réelles des locuteurs, pratiques évaluées par un sociolinguiste et non pas par les locuteurs euxmêmes comme c'est le cas dans la majorité des études ${ }^{13}$. Filhon (2009) note cependant que, même si elle n'est pas effectivement transmise, la langue revêt pour les enfants une forte valeur symbolique. Pour Billiez (1985), la tension entre les représentations et les pratiques lan-

des objectifs des ELCO qui était de préparer les enfants de migrants à un retour définitif au pays. Si le retour au pays n'est plus aussi souvent concrétisé à l'heure actuelle, il est très présent à l'esprit de beaucoup d'élèves, sans que l'on puisse déterminer s'il s'agit d'un réel désir ou d'une simple affirmation.

12. Voir aussi Filhon (2009) qui démontre que l'arabe dialectal est peut-être la première langue parlée aux enfants, mais pas obligatoirement celle qu'ils maitrisent le mieux.

13. On peut en effet s'interroger sur les résultats des enquêtes comme celle publiée par Akinci et coll. (2004) à partir d'un questionnaire adressé à des élèves du primaire, entre 6 et 11 ans, qui ne sont pas forcément les mieux placés pour évaluer leurs compétences en langue. Qu'ils affirment par exemple lire l'arabe : s'agit-il réellement de déchiffrer les lettres ou de comprendre un texte? Qu'ils disent comprendre l'arabe : serait-ce dans des situations familiales uniquement ou cela dénote-t-il une réelle maitrise de la langue? Barontini (2008) déplore qu' «on manque de données empiriques fines tout autant que d'éléments statistiques à grande échelle pour une meilleure prise en compte des pratiques effectives» (p. 108). 
gagières, renforcée par des situations sociolinguistiques conflictuelles, dissuade les jeunes de parler arabe. De moins en moins dialectophones, les élèves expriment souvent en classe la culpabilité linguistique dont il a été question plus haut : ils devraient déjà parler arabe et se retrouver en classe peut, pour certains d'entre eux, mettre en évidence une souffrance qu'ils taisent bien souvent. Plusieurs études ont porté sur la dévalorisation des connaissances linguistiques des descendants de migrants, montrant le «gâchis sur le plan personnel et sociétal que représente le fait de les considérer comme des individus sans culture et sans langue» (Billiez, Biichlé \& Abouzaïd, 2012, p. 305), image que leur renvoient bien souvent les locuteurs natifs du monde arabe que ce soit en France et surtout dans le pays de leurs origines. Or, le professeur peut s'appuyer sur ces acquis langagiers extra-scolaires pour revaloriser l'élève et l'aider à faire le lien entre l'arabe appris en classe et ce qu'il connait de son dialecte.

\subsubsection{L'Enseignement de langue et culture d'origine (ELCO)}

En France, on estime à environ 40000 les élèves qui apprennent l'arabe à l'école primaire par le biais de l'Enseignement des langues et cultures d'origine. Les cours d'arabe, avec les communications familiales et un retour régulier au pays, font partie des facteurs aidant à la retransmission de la langue (Filhon, 2009). Mais dans son rapport intitulé L'immigration à l'école de la République, Berque (1985) dénonce les effets pervers de certaines actions destinées aux enfants de migrants, qui ne font parfois que renforcer leur statut d'enfants «différents» au lieu de les amener à s'épanouir en acceptant les deux cultures. Billiez (2002) cite par ailleurs divers travaux qui ont montré que l'enseignement de l'arabe comme langue de culture et d'origine à l'école primaire a davantage posé de problèmes, en renforçant les différences linguistiques et identitaires, qu'il n'a été pertinent. Parmi les problèmes de l'ELCO arabe, elle cite notamment les «dérives des actions interculturelles vers la folklorisation de la culture dont on peut redouter qu'elles n'entretiennent ou renforcent des stéréotypes [et la] noncoïncidence, enfin, entre la langue cible et les vernaculaires parlés et/ou compris par les enfants» (p. 90). Ces différentes études concluent sur un enseignement souvent contreproductif en cela qu'il conduit parfois à la dévalorisation de l'enfant, à la dépréciation de sa famille, de ses connaissances linguistiques et culturelles, sans pour autant lui apprendre à communiquer dans la langue objet du cours. Si nous pouvons aussi observer ce sentiment dans le secondaire, devant l'ampleur 
du chemin à parcourir, ce constat devrait plutôt amener les professeurs d'arabe à discuter de stratégies qu'il serait envisageable de mettre en place.

Lors du passage au collège, pour ces élèves qui ont déjà suivi les cours d'ELCO, un autre problème va générer un climat pesant : celui du passage des cours de type «religion et tradition» de l'ELCO à des cours de langue étrangère dans un cadre laïc. Le professeur va tenir un discours parfois opposé à celui auquel l'élève avait auparavant été confronté ${ }^{14}$. Le cadre même de l'enseignement de l'arabe dans le secondaire, à savoir l'enseignement d'une langue vivante étrangère intégré à une éducation laïque, va parfois en lui-même générer des réactions fortes, du fait du conflit de discours et de méthodes entre les habitudes prises en ELCO, dans les mosquées ou dans les milieux associatifs et celles qu'exigent un enseignant certifié ou agrégé laïc, professionnel formé à l'enseignement de l'arabe. Une concertation entre intervenants en ELCO et professeurs du secondaire suffit parfois à prévenir ce type de réactions de la part des élèves auxquels on peut expliquer les différences entre les deux types d'enseignement.

\subsection{Les émotions du professeur d'arabe}

Les élèves, s'ils tiennent une place centrale, évidemment, ne sont pas les seuls acteurs en classe : le professeur y est déterminant. Il peut lui aussi ressentir diverses émotions. Sa passion pour la langue et pour l'enseignement, la joie et le plaisir qu'il tire de «passer» la langue aux élèves, n'occultent pas d'autres émotions, préjudiciables au travail en classe, en particulier l'exaspération qu'il peut éprouver vis-à-vis de situations matérielles et politiques ${ }^{15}$ qu'il juge immorales ${ }^{16}$ ou face aux

14. Nous devrions dire auquel il est toujours confronté, car beaucoup de nos élèves poursuivent les cours d'arabe à la mosquée ou dans le milieu associatif. Le poids réel de l'enseignement en milieu associatif reste à évaluer, mais il explique en partie le désintérêt croissant des familles pour l'ELCO (Petek, 2004).

15. Si les langues sont officiellement sur un pied d'égalité, l'enseignement de l'arabe pâtit d'un désengagement politique. Sinon, comment expliquer que des 40000 élèves qui apprennent l'arabe en primaire, seuls quelques milliers vont continuer au collège et au lycée, là où le chinois est passé de 2000 à 25000 élèves en quelques années (Ministère de la Culture, 2011)?

16. D'après notre propre expérience, d'un établissement à un autre, cette stigmatisation peut aller de simples vexations (une autorisation de photocopies moindre que celle des autres collègues de langue; la salle de cours attitrée 
préjugés de certains collègues. Il peut être peiné du statut informel de l'arabe, langue socialement dévalorisée (Billiez \& Trimaille, 2002). En effet, même si officiellement l'arabe est proposée comme langue étrangère au même niveau que l'allemand, l'espagnol, l'italien ou le chinois, force est de constater que les élèves des classes d'arabe forment un microcosme, une «classe-ghetto» renvoyant aux élèves une image très stigmatisante et souvent fort dépréciative (Varro, 1992, 1999; Lambert \& Trimaille, 2012). Le professeur peut aussi être décontenancé par ses élèves lorsqu'il prend la mesure de l'image qu'ils ont de la langue arabe et de la culture qu'elle véhicule. Le professeur peut aussi souffrir de classes-ghettos où les problèmes familiaux et socioéconomiques sont réels et certainement plus nombreux que dans une autre classe.

Billiez et Trimaille (2002) s'interrogent à juste titre sur la complexité de la planification linguistique de l'arabe $^{17}$ et affirment que l'enseignement d'une langue minoritaire et minorisée ne peut pas être calqué sur celui des langues socialement valorisées.

\subsection{Le cumul des situations problématiques}

Nous avons esquissé plus haut les facteurs qui, selon nous, font de l'enseignement de l'arabe un enseignement complexe. Tous n'expliquent pas ce qui peut amener un élève à refuser d'apprendre l'arabe,

qu'on donne un trimestre entier à une formation extrascolaire, obligeant le professeur à chercher une salle avec ses élèves, etc.) à des obligations pédagogiques aberrantes (voir note 17).

17. «La planification linguistique, au sein du système éducatif, représente un lieu où diverses articulations se complexifient singulièrement : entre les décisions politiques et, sur le terrain, les acteurs [...], entre les statuts formel et informel des langues, entre curriculum réel et curriculum caché (les choix de langues effectués par les consommateurs avertis d'école servent bien d'autres objectifs que la maitrise d'un autre système linguistique), entre respect de droits fondamentaux (celui de pouvoir étudier sa langue) et ses effets pervers, entre autonomie et ingérence pédagogique, entre la langue standardisée enseignée et les pratiques langagières (ou entre l'écriture et l'oralité, ou encore entre mono-, bi- et plurilinguisme), entre les résistances aux changements pédagogiques et les progrès des recherches en sociolinguistique et didactique des langues, entre pesanteurs et dynamiques, etc. C'est alors peu dire que l'abord de ce type de problématique plonge parfois le chercheur dans le désarroi, surtout lorsque aucune des mesures, successivement envisagées par l'institution éducative, ne paraît construire et favoriser le multilinguisme des enfants issus des migrations.» (p. 109) 
mais forment un terrain propice où d'autres situations vont favoriser ce décrochage. Parfois, l'élève qui apprend l'arabe se voit confronté à une langue qu'il pense inaccessible, qu'il n'a souvent pas choisi d'apprendre et qui le renvoie de manière violente à son histoire familiale tout en le stigmatisant encore davantage, dans un contexte social loin d'être évident à vivre. Ce n'est évidemment pas la situation que connaissent tous nos élèves : dans certaines classes, ces difficultés sont répandues, dans d'autres quasi inexistantes. L'enseignant d'arabe, avant même de transmettre une langue, doit aider ces élèves à gérer leur double culture, à trouver une autre réaction au poids de la stigmatisation que celui du refus de la langue.

Nous avons pu constater qu'il arrive très fréquemment qu'un élève refuse en bloc la langue arabe, parce que celle-ci est émotionnellement trop liée à son histoire personnelle. L'adolescence arrivant, abandonner l'arabe est une manière d'exprimer son refus du poids de la tradition ou de la religion dans sa famille, son mal-être vis-à-vis de sa double culture, la stigmatisation supplémentaire que produit le fait d'apprendre l'arabe ${ }^{18}$, etc. La relative ghettoïsation de la classe d'arabe n'est pas sans conséquence dans ce processus, sans compter que souvent, l'élève est inscrit de manière forcée par les parents, pour des raisons essentiellement religieuses d'après ce que nous avons pu constater. Il est évident que la composition des classes d'arabe n'est pas sans influence sur la dynamique du groupe. Pour la plupart, elles constituent ce que SaintJacques (2000) a nommé des «milieux soustractifs». Au-delà de 40\% d'élèves de milieu défavorisé, le milieu «soustractif» rend l'enseignement très difficile à gérer et provoque une augmentation considérable du nombre d'élèves en situation d'échec ou d'abandon de l'école. Terrisse et Lefebvre (2007) ajoutent que les tensions interethniques aggravent encore cette situation. Que devrait-on alors dire de nos classes d'arabe?

Loin de nous ici l'idée de dresser un constat macabre. Une fois la situation considérée, il faut rechercher les manifestations de ce refus en classe, le détecter pour y remédier rapidement. Le refus d'apprendre

18. Pour preuve de cette stigmatisation, le fait que des membres de l'administration scolaire acceptent qu'un élève qui n'a jamais appris l'arabe intègre le groupe de $2^{\text {de }}$ ou de $1^{\text {re }}$ LV1-LV2, du simple fait qu'il ait un nom à consonance arabe, ce qu'on n'imaginerait pas dans les autres langues. Le fait qu'un élève primo-arrivant soit placé d'office en arabe alors qu'il préfèrerait poursuivre la langue qu'il avait commencé à apprendre dans son pays d'origine en est un autre exemple. 
l'arabe peut être clairement, verbalement énoncé au professeur par certains élèves. D'autres, sans admettre directement ce refus, le montrent matériellement en n'ayant pas de cahier, ou par leur attitude en classe en refusant de noter le cours, en ne faisant aucun exercice, aucun devoir, en rendant systématiquement copie blanche. Si beaucoup de ces élèves semblent garder leur refus pour eux, d'autres, par des réflexions déplaisantes continuelle ${ }^{19}$, tentent ostentatoirement de décourager l'ensemble de la classe. Un découragement qui gagne facilement l'ensemble de ce microcosme qu'est la classe d'arabe, à moins que l'enseignant vigilant parvienne à y mettre un terme.

\section{Analyse du processus et propositions}

Quand l'élève se laisse submerger par des émotions négatives, il entre dans un cercle vicieux qui le peut le conduire, petit à petit ou brutalement, à abandonner l'arabe. Malheureusement, aucune étude, à notre connaissance, n'a porté sur ce phénomène en particulier. Finalement, les études se placent en général soit sous le signe du lien entre langue, immigration et intégration (Filhon, 2009; Varro, 1992, 1999), soit du point de vue de la reconnaissance de l'arabe maghrébin comme langue de France (Caubet, 2003; Barontini, 2013). Nous formulons le vœu que cette première analyse puisse initier une étude centrée sur le ressenti de l'élève en situation d'apprentissage de l'arabe. Ici, nous allons étudier le mécanisme qui se met en route de manière à mieux le comprendre et à proposer des points d'intervention où le professeur peut briser le cercle.

\subsection{Les prétextes évoqués par l'élève pour justifier son abandon de la langue}

Une première solution envisageable va être de s'attaquer aux prétextes qu'énoncent les élèves pour justifier leur comportement. On part alors du principe que l'élève va accepter de raisonner de bonne foi sur ses propres propos, en suivant l'argumentation du professeur.

19. Parmi les incontournables : l'arabe est une langue difficile; l'arabe ne sert à rien; le professeur précédent était beaucoup mieux; cet exercice n'a aucun intérêt... On observe aussi de nombreuses remarques concernant les supports (les illustrations ne sont pas belles, l'histoire est sans intérêt, la chanson ne plait pas...). 


\subsection{1. "L'arabe est une langue difficile »}

C'est le prétexte qui est censé tout excuser. Or, la difficulté d'une langue est plus liée à l'amour et à la volonté d'apprendre qu'à de réelles difficultés techniques. Pour Romero-Muñoz (2011), «le degré de difficulté dépend aussi - peut-être même essentiellement - de facteurs affectifs. L'amour, la haine, la solitude peuvent vous pousser à apprendre une langue ou, au contraire, nous faire développer une aversion épidermique pour celle-ci» (p. 20).

Il est vrai qu'il y a des raisons dans l'histoire des élèves qui expliquent que l'arabe a une image de langue difficile, notamment la confrontation de leurs pratiques langagières avec des locuteurs natifs, en général lorsqu'ils vont en vacances dans le pays d'origine de leurs parents. Ils sont souvent moqués pour leur accent et leurs erreurs. Billiez (1985) montre comment la dévalorisation du dialecte, renforcé par la mythification de la «vraie» langue arabe, place les élèves dans un état défavorable à l'apprentissage où ils se jugent eux-mêmes inaptes à parler, et donc à apprendre l'arabe, bien que cette langue ait pour eux une valeur hautement symbolique. L'arabe enseigné en classe va être réputé difficile et l'élève, s'il en a, ne va pas s'appuyer sur ses acquis qu'il auto-dévalorise à la suite des réflexions qu'on a pu lui faire. Le refus de prendre la parole est un signe auquel le professeur doit attacher de l'importance, car il peut tout à fait révéler une insécurité linguistique ${ }^{20}$ (la peur de mal parler, la colère de ne pas savoir parler «sa» langue) qui peut l'amener à démissionner.

\subsection{2. "Je ne comprends rien, c'est dur»}

Cette affirmation, qui découle de la précédente, illustre bien le blocage psychologique que font certains élèves vis-à-vis d'une langue présentée comme difficile. Il s'agit aussi d'un phénomène généralisé, une habitude mentale consistant, avant même d'avoir lu l'énoncé d'un exercice et d'y avoir un peu réfléchi, de décréter que c'est dur et qu'on n'y comprend rien. En multipliant les tâches qui génèrent des émotions positives (plaisir de réussir, d'avoir compris et retenu), le professeur peut amener l'élève à prendre conscience qu'il possède des connais-

20. À propos de l'insécurité linguistique liée à la langue arabe, voir notamment Calvet (1999) et Larcher (2008) pour le décryptage d'un ouvrage majeur pour comprendre ces questions du rapport des locuteurs de l'arabe à la langue arabe, écrit par Choubachy traduit en 2007 par Le Sabre et la virgule. La langue du Coran est-elle à l'origine du mal arabe? (Paris : l'Archipel). 
sances suffisantes pour réussir la tâche donnée. Il a en effet été prouvé que les capacités de mémoire dépendent de l'état émotionnel de l'apprenant (Blanc, 2006) et surtout que, lorsqu'on atteint facilement un but, le sentiment de plénitude qui s'en suit nous amène à considérer que l'ensemble de nos connaissances nous a permis de réussir. À l'inverse, quand on échoue, la déception voire l'abattement amène l'élève à considérer qu'il ne sait rien ou que ce qu'il sait ne lui sert à rien. On doit donc veiller à proposer des activités qui confortent l'élève dans son apprentissage et l'encouragent à le poursuivre. On touche ici au sentiment d'efficacité personnelle et à la motivation de l'élève, élément moteur fondamental dans son apprentissage (Puozzo Capron, 2009, 2012), qu'on peut l'aider à développer.

\subsection{3. "Je ne sais pas lire»}

$\mathrm{Si}$, à l'oral, beaucoup d'élèves refusent de parler, ils sont aussi nombreux à décréter qu'ils ne savent pas lire ni écrire. Dans leur esprit, il s'agit d'une lacune rédhibitoire qui justifie leur abandon de l'apprentissage de cette langue. Mais cette position de principe a de réelles répercussions, car après un tel choix, l'élève va avoir tendance à modifier ses attitudes de manière à être en cohérence avec ce qu'il énonce (Festinger, 1957). Les élèves qui déclarent ne pas savoir lire une fois qu'ils ont décidé de ne plus apprendre l'arabe entrent dans ce phénomène des prédictions qui se vérifient d'elles-mêmes (Watzlawick, 1996). Il est important de prendre conscience de ces processus, tant pour l'enseignant que pour l'élève, de manière à pouvoir mettre en place des stratégies permettant de les inverser ou de les annuler.

Des habitudes en classe peuvent être prises pour amener chaque élève à prendre régulièrement la parole, mais il est plus difficile de répondre à un élève qui déclare ne pas savoir lire. Certes, l'arabe utilise un système graphique différent du français, et les tâches de déchiffrage et de lecture-compréhension peuvent paraitre difficiles aux élèves débutants. Notre propre expérience nous a amenée à développer une méthode ${ }^{21}$ qui amène l'élève à comprendre ce qu'il lit et à acquérir des

21. Nous utilisons une méthode en trois temps : apprentissage des fondamentaux de la langue en transcription, apprentissage rapide de l'alphabet et poursuite des apprentissages en écriture arabe. Ainsi, lorsqu'il apprend à écrire, l'élève écrit tout de suite des énoncés qu'il comprend. Il n'est pas confronté à des mots écrits en arabe tant qu'il ne possède pas les moyens de les lire. Nous pensons que la méthode consistant à confronter l'élève au texte arabe alors 
automatismes de lecture. On peut aussi jouer sur l'affectif, en donnant comme objectif la lecture oralisée d'un texte, en montrant aux élèves combien cette tâche est un aboutissement qui permet de montrer ses progrès en langue et d'impressionner son auditoire, de faire la fierté de ses parents.

\subsection{Rôle des émotions dans le processus de refus de la langue}

On sait que la rémanence des émotions crée une sorte de cercle vicieux :

Des pensées liées à la situation émotionnelle se manifestent au cours des heures, des jours, des semaines, voire des mois qui suivent cette situation. Elles se développent de manière principalement automatique et prennent la forme d'une rumination mentale, de pensées intrusives ou d'images mentales récurrentes. (Rimé \& Herbette, 2003, p. 71)

Ainsi, pour un élève qui a décidé d'abandonner l'apprentissage de l'arabe, chaque cours et chaque confrontation à la langue accentuent ses émotions négatives. Il trouve la langue de plus en plus difficile, il comprend de moins en moins, connait toujours l'alphabet mais ne sait plus lire, etc., mais surtout, la rancœur vis-à-vis de sa situation, dont il peut tenir responsable sa famille ou ses professeurs, ou encore les élèves qui apprécient le cours d'arabe, va s'accentuant. Pour Billiez et Trimaille (2002),

les élèves concernés vivent cette situation comme autant de violences symboliques (au sens de Bourdieu), qu'ils n'ont dès lors de cesse de vouloir retourner aux agents ou objets emblématiques de la domination, les plus accessibles étant leurs enseignants et les camarades qui n'appartiennent pas au groupe. (p. 118)

Ces phénomènes, bien connus, sont pour certains à l'origine de la violence juvénile.

Il est donc fondamental de briser le cercle vicieux dans lequel s'est installé l'élève, non pas pour sauvegarder à tout prix son apprentissage de l'arabe, mais pour lui apprendre à gérer ses émotions et à s'adapter aux situations qui lui déplaisent. Pour cela, il est essentiel de maintenir le dialogue avec l'élève à ce sujet, mettre en mots l'ensemble du processus, en partant du principe que son intelligence va être réceptive aux

que le système graphique n'est pas acquis l'amène à se trouver dans une attitude d'incompréhension face aux énoncés en arabe, qui peut, à la longue, lui être préjudiciable. 
raisonnements que nous lui exposerons. Par certaines tâches, l'enseignant peut aussi guider les raisonnements de l'élève pour l'amener à relativiser son apprentissage. Nous avons vu comment l'émotion peut induire le raisonnement, mais Caverni (Channouf \& Rouan, 2002) part du principe que le raisonnement aussi influence l'émotion. Parmi les types de raisonnement qu'il énumère, on en retrouve certains chez nos élèves, comme l'attribution causale (les responsables de la situation, ce sont les parents qui ont forcé l'élève à aller en classe d'arabe, ou encore le professeur précédent qui agissait de telle manière), le raisonnement contrefactuel (si seulement je n'avais pas choisi l'arabe, si seulement je pouvais arrêter l'arabe, etc.) ou encore la recherche de confirmations (je n'aurais pas dû être en classe d'arabe, la preuve : c'est une langue difficile, je ne sais pas lire, je ne comprends rien, etc.) qui correspond aux phénomènes des prédictions qui se vérifient d'elles-mêmes vu plus haut, encore appelées «prophéties auto-réalisatrices».

\subsection{Quelles stratégies l'enseignant peut-il mettre en place? Propositions}

Nous avons déjà évoqué quelques propositions pour gérer les émotions négatives qui surgissent en cours d'arabe, de manière à ce qu'elles ne prennent pas le pas sur les émotions positives à même de soutenir l'intérêt et la motivation de l'élève pour l'arabe. Il faut avant tout savoir reconnaitre un élève qui entre dans le cycle de refus de l'arabe. Dans l'idéal, il faut aussi en comprendre les causes, mais concrètement, en classe, quelles stratégies l'enseignant peut-il mettre en place pour éviter qu'un élève arrive au stade ultime où il refuse totalement d'apprendre quoi que ce soit, au point de n'avoir ni cahier, ni manuel, de refuser de copier le tableau ou de faire les contrôles ${ }^{22}$ ?

\subsubsection{Position de principe et rhétorique}

Nous pensons qu'il faut systématiquement et collectivement lutter contre les prétextes que prennent les élèves en démontrant que l'arabe

22. Une formation pratique en psychologie aiderait très certainement les enseignants à améliorer leurs pratiques pédagogiques, d'après Englander (2007), d'autant plus que ces difficultés se retrouvent dans toutes les disciplines. L'arabe n'est pas la seule matière où des élèves «décrochent» à ce point, mais certainement l'une de celles où l'on en dénombre proportionnellement le plus. 
n'est pas aussi difficile que certains le prétendent, en développant une méthode pour apprendre à lire en comprenant, en chargeant les apprentissages émotionnellement mais positivement, en pratiquant ce qu'on pourrait nommer «l'autosuggestion de groupe» : partir du principe que l'arabe est une langue qu'on peut facilement apprendre et le prouver dès que l'occasion se produit. Pour cela, le professeur s'efforce d'orchestrer les différents paramètres de l'enseignement, en particulier ceux qui dépendent de lui, directement ou indirectement (Defays, 2003). Il doit proposer aux élèves des activités pédagogiques leur permettant de prendre conscience qu'ils peuvent surmonter les difficultés linguistiques propres à cette langue et même prendre plaisir à le faire.

Le professeur peut lutter contre les stéréotypes, reprendre les idées reçues de ses élèves, car elles peuvent gêner l'apprentissage. Il doit se préparer aux réactions que son enseignement va provoquer et savoir comment y répondre. Pour notre part, nous faisons tomber beaucoup de préjugés et mettons longuement les choses à plat, lors du tout premier cours, puis chaque fois que cela s'avère nécessaire. Par exemple, nous insistons sur le fait que l'arabe littéral moderne est une langue vivante qui évolue et que la langue enseignée n'est pas l'arabe classique ni la langue du Coran; que les dialectes sont de véritables langues qui existaient avant l'arabe classique; que l'arabe n'est pas difficile à apprendre, mais qu'il faut passer dans un système sémitique très différent du nôtre, etc. Nous sommes surtout attentive à toutes les remarques d'ordre dépréciatif sur la langue ou les pratiques langagières des élèves et à toute idée reçue sur la langue (difficulté, perfection, beauté, caractère saint, etc.), idées que nous recontextualisons pour les décoder et amener les élèves à les nuancer. De la même manière, nous essayons d'ouvrir au maximum le cours sur l'ensemble du domaine arabe pour faire prendre la mesure aux élèves de sa richesse et de sa diversité, et de la place qu'y occupe la langue arabe ${ }^{23}$.

23. Que tous les Arabes ne soient pas musulmans et que tous les musulmans ne soient pas Arabes est une découverte pour beaucoup d'élèves. Que l'alphabet arabe puisse être utilisé par d'autres langues, que l'arabe puisse être transcrit, et surtout que l'arabe ne soit pas la seule langue parlée dans le monde arabe, toutes ces données sont souvent nouvelles et difficiles à concevoir pour certains élèves. 


\subsubsection{Communication : lutter contre les stéréotypes et les représentations à tous les niveaux}

Il est nécessaire de communiquer sur la langue à tous les niveaux. Avec les élèves, évidemment, communiquer sur leur histoire et leur ressenti est primordial. Il faut mettre à nu ce processus de rejet de la langue et décider, ensemble, de le changer.

Il est aussi fondamental de communiquer avec les parents et l'ensemble de l'équipe éducative. Les manifestations du type «école des parents», où le professeur peut, en présence des élèves et de leur famille, montrer aux parents comment ils peuvent suivre le travail de leur enfant et l'aider dans son apprentissage de la langue, sont très utiles. C'est d'autant plus important que bon nombre de parents, qui ne connaissent pas l'arabe littéral et sont pour certains analphabètes, ne savent pas comment aider leur enfant et ont tendance, involontairement, à les encourager vers un échec, en répétant que l'arabe est une langue difficile ou en excusant l'enfant sur le fait que, s'il n'arrive pas à apprendre l'arabe, c'est que ses parents ne peuvent pas l'aider. Il est donc primordial de rencontrer les parents, de les déculpabiliser si nécessaire et de valoriser leur rôle et leurs compétences (Deslandes, 2007), tout en les amenant à prendre conscience que ce qu'ils disent de la langue arabe peut positivement comme négativement influencer l'apprentissage de leur enfant.

Auprès des élèves, des parents et des collègues, il est essentiel de faire évoluer les représentations, faire changer les attitudes, expliquer quelles sont les problématiques liées à l'enseignement de l'arabe et montrer comment on peut améliorer la situation.

\subsubsection{Didactique et méthodologie}

C'est surtout dans ses pratiques de classe que l'enseignant peut améliorer les choses. La méthodologie parait être une clé pour faire progresser les élèves. En détaillant les étapes suivies en cours et en fixant aux élèves des objectifs clairs et réalistes ${ }^{24}$, on amène l'élève à considérer son apprentissage et on favorise une bonne réception par la sécurité que procure le fait de savoir pourquoi on se fixe tels objectifs et comment on les atteint. Il faut aussi adapter les évaluations de manière

24. Une fiche présentant les différents savoirs à acquérir et objectifs à atteindre peut être distribuée aux élèves pour qu'ils prennent conscience de leurs connaissances et progrès. 
à éviter au maximum de mettre les élèves en échec. Par exemple pour le lexique, le temps scolaire n'étant pas assez long pour mémoriser totalement tous les mots ou expressions que l'on cible, on évalue dans les deux sens : on demande la traduction arabe d'un mot français quand celui-ci a suffisamment été vu pour être mémorisé, on demande la traduction française d'un mot arabe moins important ou moins vu, que l'apprenant reconnait sans pour autant arriver à le trouver directement dans la langue cible. Il convient aussi de laisser la place aux connaissances de l'élève, de lui montrer comment ces dernières peuvent l'aider à apprendre l'arabe littéral ${ }^{25}$.

Enfin, l'enseignant est invité à revaloriser la langue, en s'appuyant sur la langue même et en provocant chez les élèves des émotions fortes ayant des répercussions positives : lier l'apprentissage de la graphie à l'art de la calligraphie, faire porter l'attention sur la mélodie de la langue (due à son système morpho-phonologique), sur tous les savoirs, profanes ou sacrés, qu'elle a véhiculés et qu'elle continue de transmettre. De la même manière, il peut éviter en cours d'aborder ce qui déclenche des réactions émotives qui nuisent à l'apprentissage.

\section{Conclusion}

Les émotions jouent un grand rôle dans l'enseignement de l'arabe, pour des raisons tout à la fois culturelles, religieuses, historiques et sociales. Le professeur d'arabe doit absolument en tenir compte et composer avec cette profusion de sentiments et d'émotions, dont beaucoup s'avèrent néfastes et destructeurs et amènent un nombre important d'élèves à abandonner l'arabe. L'enseignant n'a pas la possibilité de modifier les causes de cet état de fait, mais en réfléchissant à sa relation aux élèves, à sa manière de présenter la langue arabe et en communicant sur cette dernière, en luttant contre les préjugés, il peut améliorer les choses. Sa principale marge de manœuvre, puisqu'il ne peut pas s'attaquer aux causes brutes, est d'intervenir dans les autres étapes de ce cercle vicieux : au niveau du discours des élèves, quand les premiers signes d'un refus apparaissent ou qu'ils évoquent des prétextes censés justifier un abandon de la langue; au niveau de la représentation des parents,

25. Dès que possible, nous conseillons de faire le lien avec le dialecte, de relever chaque fois que les structures sont similaires ou proches pour donner aux élèves des techniques pour qu'ils se souviennent de ce qui diffère. 
des élèves ou encore de l'équipe éducative. La communication est certainement l'une des clés pour que l'enseignement de l'arabe se décomplexe et se décharge de toutes ces émotions : communication entre le professeur et les élèves et parents d'élève, mais aussi des professeurs entre eux. Espérons que ces observations, tirées de notre expérience d'enseignante de langue arabe, impulsera des recherches plus précises qui permettront d'émettre des propositions pour dédramatiser l'enseignement de l'arabe et s'acheminer vers un apprentissage plus serein de cette langue. À défaut d'une réelle ouverture de notre discipline à l'ensemble des élèves, même si la mixité sociale apparait comme une clé essentielle pour évacuer les émotions négatives liées à l'arabe, promouvons un enseignement décomplexé et aidons nos élèves à y voir plus clair.

\section{RÉFÉRENCES BIBLIOGRAPHIQUES}

Akinci, M. A., De Ruiter, J. J. \& Sanagustin, F. (2004). Le plurilinguisme à Lyon. Le statut des langues à la maison et à l'école. Paris : l'Harmattan.

BarontinI, A. (2008). Radiographie sommaire des pratiques de l'arabe maghrébin en France. Hommes et migrations, 1288, 104-109.

BARONTINI, A. (2013). Locuteurs de l'arabe maghrébin-langue de France : une analyse sociolinguistique des représentations, des pratiques langagières et du processus de transmission. Thèse de doctorat, inédit.

Berque, J. (1985). L'immigration à l'école de la République : rapport au ministre de l'Éducation nationale. Paris : La documentation française, CNDP.

BilLIEZ, J. (1985). La langue comme marqueur d'identité. Revue européenne des migrations internationales, 1 (2), 95-104.

BilLIEZ, J. (2002). De l'assignation à la langue d'origine à l'éveil aux langues : vingt ans d'un parcours sociodidactique. Ville-École-Intégration Enjeux, 130, 87-101.

Billiez, J. \& Trimaille, C. (2001). Plurilinguisme, variations, insertion scolaire et sociale. Langage et société, 4 (98), 105-127.

Billiez, J., Biıchlé, L. \& AbouzAï̀, M. (2012). À la recherche de l'«arabe vrai» en situation migratoire. Dans A. Barontini \& D. Caubet (dir.), Dynamiques langagières en Arabophonies (p. 293-309). Saragosse : Université de Saragosse.

Blanc, N. (dir.). (2006). Émotion et cognition. Quand l'émotion parle à la cognition. Paris : éditions In Press. 
Calvet, L.-J. (1999). Pour une écologie des langues du monde. Paris : Plon.

Channouf, A. \& Rouan, G. (dir.). (2002). Émotions et cognitions. Bruxelles : De Boeck.

CAubet, D. (2003). La reconnaissance de l'arabe «dialectal» en France : un parcours sinueux. Dans J. Lentin \& L. Antoine (dir.), Mélanges David Cohen, Études sur le langage, les langues, les dialectes, les littératures, offertes par ses élèves, ses collègues, ses amis, présentées à l'occasion de son anniversaire (p. 135-148). Paris : Maisonneuve et Larose.

Colletta, J.-M. \& TCherkassof, A. (2003). Les émotions. Cognitions, langage et développement. Sprimont : Mardaga.

Defays, J.-M. (2003). Le français langue étrangère et seconde : enseignement et apprentissage. Sprimont : Mardaga.

Deslandes, R. (2007). Rôle de la famille, liens école-famille et résilience scolaire. Dans B. Cyrulnik \& J.-P. Pourtois, École et résilience (p. 271295). Paris : Odile Jacob.

Englander, H. (2007). Comment les enseignants peuvent-ils devenir des tuteurs de résilience? Dans B. Cyrulnik \& J.-P. Pourtois, École et résilience (p. 227-250). Paris : Odile Jacob.

Filhon, A. (2009). Langues d'ici et d'ailleurs : transmettre l'arabe et le berbère en France. Paris : Institut national d'études démographiques.

Héran, F., Filhon, A. \& Deprez, C. (2002). La dynamique des langues en France au fil du $\mathrm{xx}^{\mathrm{e}}$ siècle. Population et sociétés, 376.

LAmbert, P. \& Trimaille, C. (2012). La variation stylistique : un contenu à intégrer dans la formation des enseignants. Dans C. Balsiger, D. Bétrix Kölher, J.-F. De Pietro \& C. Perregaux (dir.), Éveil aux langues et approches plurielles. De la formation des enseignants aux pratiques de classe (p. 255-267). Paris : l'Harmattan.

Larcher, P. (2008). «Le Sabre et la virgule» de Chérif Choubachy. Compte rendu fait à Louis-Jean Calvet. Dans A. Moussirou-Mouyama (dir.), Les boîtes noires de Louis-Jean Calvet (p. 227-245).

MinistèRe DE L'ÉDUCATION NATIONALE. (2008). Le centenaire de l'agrégation d'arabe. Actes du colloque organisé par la direction générale de l'enseignement scolaire. Formation continue publications. Disponible sur <http://catalogue.crdp.ac-versailles.fr/acces_ressources/ressources/ actes_agregation_arabe_110197.pdf $>$.

Ministère de la Culture. (2011). La langue arabe en France. Disponible sur <http://www.dglflf.culture.gouv.fr/publications/Rencontres2011_ La_langue_arabe_en_France.pdf $>$.

Petek, G. (2004). Les ELCO, entre reconnaissance et marginalisation. Langues de France, 1252, 45-55.

Pinon, C. (2011). La grammaire arabe : entre théories linguistiques et applications didactiques ». Synergie Monde arabe, 7, 75-86. Disponible sur 
$<$ http://ressources-cla.univ-fcomte.fr/gerflint/Mondearabe 7/monde arabe $7 . h t m l>$.

Pinon, C. (2012). Les enjeux épistémologiques et didactiques d'une grammaire arabe fondée sur corpus. Dans T. Arnavielle (dir.), Voyages grammairiens (p. 83-101). Paris : L'Harmattan.

Puozzo Capron, I. (2009). Le sentiment d'efficacité personnelle. Pour un nouvel enseignement/apprentissage des langues. Sciences Croisées, 6. Disponible sur $<$ http://sciences-croisees.com/N6/PuozzoCapron.pdf $>$.

Puozzo CAPron, I. (2012). Le sentiment d'efficacité personnelle et l'apprentissage des langues. Les Cahiers de l'Acedle, 9 (1), 75-94. Disponible sur <http://acedle.org/IMG/pdf/05_Puozzo-Capron.pdf>.

Rimé, B. \& Herbette, G. (2003). L'impact des émotions : approche cognitive et sociale. Dans J. M. Coletta \& A. Tcherkassof, Les émotions. Cognitions, langage et développement (p. 69-84). Sprimont : Mardaga.

Romero-MuÑoz, E. (2011). Il faut en finir avec l'enseignement des langues. Namur : Presses universitaires de Namur.

Terrisse, B. \& Lefebvre, M.-L. (2007). L'école résiliente : facteurs de risque, facteurs de protection et sentiment d'efficacité collective. Dans B. Cyrulnik \& J.-P. Pourtois, École et résilience (p. 47-83). Paris : Odile Jacob.

VARRO, G. (1992). Les «langues immigrées» face à l'école française. Language Problems and Language Planning, 16 (2), 137162.

VARRo, G. (1999). Les discours officiels sur les élèves. Dans A. Paveau \& P. E. Müller (dir.), L'École en débat. Mots / Les langages du politique, 61, 49-66.

Watzlawick, P. (1996). L'invention de la réalité. Comment savons-nous ce que nous croyons savoir? Contributions au constructivisme. Paris : Points. 